\title{
Internal tension in a collapsed polymer under shear flow and the connection to enzymatic cleavage of von Willebrand factor
}

\author{
Matthias Radtke ${ }^{1, a}$, Svenja Lippok ${ }^{2}$, Joachim O. Rädler ${ }^{2}$, and Roland R. Netz ${ }^{1}$ \\ 1 Fachbereich Physik, Freie Universität Berlin, Arnimallee 14, 14195 Berlin, Germany \\ 2 Fakultät für Physik der Ludwig-Maximilians-Universität München, Schellingstraße 4, 80799 München, Germany
}

Received 17 December 2015

Published online: 22 March 2016

(c) The Author(s) 2016. This article is published with open access at Springerlink.com

\begin{abstract}
By means of Brownian hydrodynamics simulations we show that the tension distribution along the contour of a single collapsed polymer in shear flow is inhomogeneous and above a threshold shear rate exhibits a double-peak structure when hydrodynamic interactions are taken into account. We argue that the tension maxima close to the termini of the polymer chain reflect the presence of polymeric protrusions. We establish the connection to shear-induced globule unfolding and determine the scaling behavior of the maximal tensile forces and the average protrusion length as a function of shear rate, globule size, and cohesive strength. A quasi-equilibrium theory is employed in order to describe the simulation results. Our results are used to explain experimental data for the shear-sensitive enzymatic degradation of von Willebrand factor.
\end{abstract}

\section{Introduction}

The study of the dynamics of polymers in solution has become an important aspect for understanding non-equilibrium processes in biopolymeric systems [1]. In the context of blood coagulation, considerable research has been focused on the multimeric glycoprotein von Willebrand factor (VWF). The binding of VWF to exposed collagen at sites of vascular injuries and the simultaneous VWFmediated adhesion of platelets are central steps in primary hemostasis [2-4]. In this context, the presence of shear or elongational flow constitutes a crucial ingredient as it activates the functional conformation of VWF multimers by inducing a transition from globular to unfolded conformations [5-7] and thus facilitates adhesion to the extracellular matrix $[8,9]$. Previous simulation studies elucidated the dynamics and the VWF adsorption behavior $[9,10]$ and revealed that VWF must exhibit finely adjusted, longlived bonds in order to resist hydrodynamic forces and to allow for shear-induced adhesion [11,12].

The existence of shear, apart from inducing conformational changes of the VWF multimers, can presumably also influence VWF's function by changing the tertiary structure of individual monomers. In particular, it has been argued that tensile forces lead to the unfolding of individual domains [13,14] and consequent exposure of binding and cleavage sites that in equilibrium conditions

\footnotetext{
a e-mail: radtkem@zedat.fu-berlin.de
}

or at low shear rates are not accessible, e.g. due to shielding effects of VWF domains $[15,16]$.

Another shear-induced biological process in which VWF takes part is the reversible platelet aggregation under high shear rate [17-20] that has pathophysiological relevance. In fact, malfunction in the VWF degradation and the resulting high amount of abnormally long VWF multimers can lead to life-threatening thrombotic diseases $[21,22]$. The shear-dependent VWF degradation thus presents an active area of research [23]. The required regulatory mechanism is associated with the specific metalloprotease ADAMTS13, which is responsible for VWF length regulation and thereby controls the hemostatic activity $[2,24]$. Note that the length distribution of VWF in plasma has been determined recently using fluorescence correlation spectroscopy (FCS) [25]. The cleavage site at which ADAMTS13 acts is deeply buried within the VWF A2 domain in the native state [26]. There is general consensus that VWF degradation requires shear or forceinduced A2 domain opening and exposure of the cleavage site for ADAMTS13 [14, 27-29]. The force-induced activation of single A2 domains was recently studied using laser tweezers [13]. In that study, cleavage of unfolded A2 domains was observed and the dependence of the catalytic rate on the enzyme concentration was determined. Details about the underlying molecular mechanism were provided by means of molecular dynamics simulations [14].

In the present study we address the interplay between shear-flow-induced VWF unfolding and the internal 
tensile force distribution along the polymer contour, the opening of the mechanosensitive VWF A2 domain and the activity of the cleavage enzyme ADAMTS13. We use a coarse-grained VWF polymer model and employ Brownian hydrodynamics simulations. In the first part, we show that the shear-induced unfolding transition of a collapsed globule, where elongated configurations are periodically observed for short time intervals, can be connected to an inhomogeneous profile of the average tension between beads along the polymer chain. The unfolding has previously been explained by a nucleation model based on the existence of thermally activated polymeric protrusions [5]. Here, we present a direct proof of the existence of such protrusions and corroborate the protrusion-induced instability mechanism leading to unfolding of collapsed polymers in shear flow. We study the tension profile as a function of shear rate, chain length, and cohesive strength. The average maximal force (the peak force) along the chain contour is identified as the typical force acting on a protrusion. We determine scaling relations for the dependence of the peak force and the average protrusion length on the size of the globule and its cohesive strength. Using these scaling predictions, we develop a quasi-equilibrium theory to describe the average length of protrusions.

In the second part we show how the shear-induced tension profile along the polymer is connected to the physiological process of shear-dependent proteolysis of VWF by its specific cleavage enzyme ADAMTS13. We formulate a simple stochastic two-state model for the VWF A2 domain opening and calculate the force-dependent probability for the domain to be accessible to cleavage by ADAMTS13 from the tension distribution along our coarse-grained polymer model of VWF. The result can be connected to the shear-dependent cleavage rate of full-length VWF mediated by ADAMTS13 that has been recently measured experimentally by Lippok et al. [30]. In that study, fluorescence correlation spectroscopy (FCS) was employed in combination with a microfluidic shear cell to quantify the effect of shear on the kinetics of VWF cleavage in aqueous buffer and in blood plasma by measuring the time-dependent increase in VWF multimer concentration. Our theoretical modeling allows to deduce characteristic parameters of single cleavage sites like the effective force scales of the stochastic opening and closing processes. We compare our results with laser tweezer experiments [13] on single A2 domains and argue that the domain opening in shear flow might not be equivalent to the domain unfolding probed by external stretching forces.

\section{Simulation method}

Brownian hydrodynamics simulations are performed using the discretized Langevin equation

$$
\begin{aligned}
& \mathbf{r}_{i}(t+\Delta t)-\mathbf{r}_{i}(t)= \\
& \left(\boldsymbol{\mu}_{i i} \dot{\gamma} z_{i} \hat{\mathbf{x}}-\sum_{j=1}^{N} \boldsymbol{\mu}_{i j} \cdot \nabla_{\mathbf{r}_{j}} U\left(\left\{r_{N}(t)\right\}\right)\right) \Delta t+\boldsymbol{\xi}_{i}(\Delta t),
\end{aligned}
$$

which describes the displacement of bead $i$ at position $\mathbf{r}_{i}$ after the time step $\Delta t$. Note that all quantities used are made dimensionless by rescaling lengths $r=\tilde{r} / a$ by the bead radius $a$, energies $U=\tilde{U} / \mathrm{kT}$ by thermal energy and times $t=\tilde{t} / \tau$ by the characteristic diffusion time $\tau=$ $a^{2} / \mu_{0} \mathrm{kT}=6 \pi \eta a^{3} / \mathrm{kT}$, where $\eta$ is the viscosity. The first term in eq. (1) represents a linear shear flow with rate $\dot{\gamma}=$ $\tilde{\dot{\gamma}} \tau$, where $\hat{\mathbf{x}}$ is the unit vector in $x$-direction. The second term accounts for the direct force acting on particle $i$ itself as well as the hydrodynamic flow field created by forces acting on all other particles $j \neq i$. Hydrodynamic interactions are taken into account via the mobility matrix approximated by the Rotne-Prager-Yamakawa tensor [31, 32]

$$
\begin{aligned}
& \boldsymbol{\mu}_{i j}=\tilde{\boldsymbol{\mu}}_{i j} / \mu_{0}= \\
& \left\{\begin{array}{l}
\frac{3}{4 r_{i j}}\left(\left(1+\frac{2}{3 r_{i j}^{2}}\right) \mathbf{1}+\left(1-\frac{2}{r_{i j}^{2}}\right) \frac{\mathbf{r}_{i j} \mathbf{r}_{i j}}{r_{i j}^{2}}\right), \quad r_{i j} \geq 2, \\
\left(1-\frac{9 r_{i j}}{32}\right) \mathbf{1}+\frac{3 r_{i j}}{32} \frac{\mathbf{r}_{i j} \mathbf{r}_{i j}}{r_{i j}^{2}}, r_{i j}<2,
\end{array}\right.
\end{aligned}
$$

where $\mathbf{r}_{i j}=\mathbf{r}_{i}-\mathbf{r}_{j}$ and $r_{i j}=\left|\mathbf{r}_{i j}\right|$; self-mobilities are given by $\boldsymbol{\mu}_{i i}=\tilde{\boldsymbol{\mu}}_{i i} / \mu_{0}=\mathbf{1}$. For free draining simulations the off-diagonal elements of the mobility matrix eq. (2) are set to zero. The stochastic contribution $\boldsymbol{\xi}_{i}$ is given by Gaussian random vectors with correlations according to the fluctuation-dissipation theorem $\left\langle\boldsymbol{\xi}_{i} \boldsymbol{\xi}_{j}\right\rangle=2 \boldsymbol{\mu}_{i j} \Delta t$ and vanishing mean. The simulation typically runs for $10^{8}$ time steps of length $\Delta t=5 \times 10^{-4}$.

The homopolymer model consists of $N$ beads, which interact via Lennard-Jones potentials of depth $\varepsilon=\tilde{\varepsilon} / \mathrm{kT}$ and are connected in a linear chain by stiff bonds with a rescaled spring constant $\kappa=\tilde{\kappa} a^{2} / \mathrm{kT}=200$; the total potential reads

$$
U=\varepsilon \sum_{i<j}\left(\left(2 / r_{i j}\right)^{12}-2\left(2 / r_{i j}\right)^{6}\right)+\frac{\kappa}{2} \sum_{i}\left(r_{i, i+1}-2\right)^{2} .
$$

In order to obtain a measure for the absolute value of the tensile force $f_{i} \equiv\left\langle f_{i}\right\rangle=\kappa\left(\left\langle r_{i, i+1}\right\rangle-2\right)$ along the bond between beads $i$ and $i+1$, the average distance $\left\langle r_{i, i+1}\right\rangle$ is recorded during the course of simulation using block averages in a time interval $t=0.05$.

\section{Results for tensile force profiles}

We consider a collapsed polymer of length $N=50$ having fixed cohesive strength $\varepsilon=2$, unless stated otherwise. The influence of an applied shear flow on the size of the globule is shown in fig. 1a. The squared radius of gyration $R_{g}^{2}=N^{-1} \sum_{i}\left(\mathbf{r}_{i}-\mathbf{r}_{\text {com }}\right)^{2}$, with $\mathbf{r}_{\text {com }}=N^{-1} \sum_{i} \mathbf{r}_{i}$, monotonically increases as a function of shear rate $\dot{\gamma}$, where we subtract $\left(R_{g}^{0}\right)^{2}=R_{g}^{2}(\dot{\gamma}=0)=11.3$, the globule radius of gyration measured at vanishing shear $\dot{\gamma}=0$. The black line indicates a scaling relation $R_{g}^{2}-\left(R_{g}^{0}\right)^{2} \sim \dot{\gamma}^{2}$ for not too large shear rates $\dot{\gamma}<40$. The conformational change of a polymeric globule in shear flow has been investigated in previous studies [5,6] reporting a quite sharp 

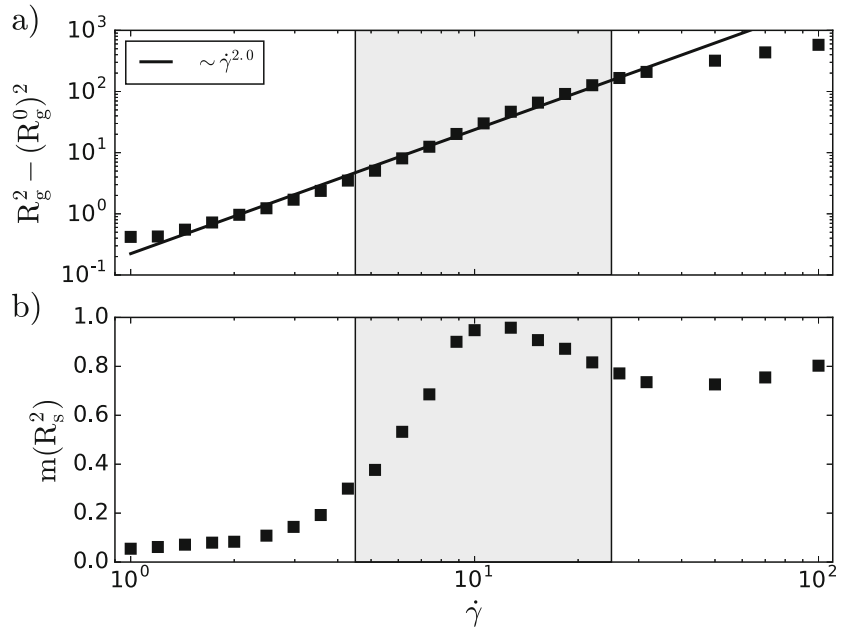

Fig. 1. a) Squared radius of gyration $R_{g}^{2}$ as a function of shear rate $\dot{\gamma}$ for cohesive strength $\varepsilon=2$ and $N=50$; hydrodynamic interactions are included. The equilibrium value $\left(R_{g}^{0}\right)^{2}=11.3$ is subtracted. The solid line represents a power law with exponent 2. b) The rescaled variance of the squared extension $m\left(R_{S}^{2}\right)$ displays a maximum at about $\dot{\gamma}^{*}=10$ corresponding to the critical shear rate of globule unfolding. The area shaded in gray indicates where the force profiles in fig. 2a exhibit a double-peak structure.

unfolding transition at a critical shear rate $\dot{\gamma}^{*}$ at which large size fluctuations are observed. In those studies, the shear-induced unfolding transition is defined by the maximum of the rescaled variance of the squared extension $m\left(R_{S}^{2}\right)$, displayed in fig. 1b. The extension $R_{S}$ is defined as the maximal distance in flow direction between any two beads from which the rescaled variance follows as $m\left(R_{S}^{2}\right)=\left(\left\langle R_{S}^{4}\right\rangle-\left\langle R_{S}^{2}\right\rangle^{2}\right) /\left\langle R_{S}^{2}\right\rangle^{2}$. We obtain for $N=50$ and $\varepsilon=2$ the critical shear rate $\dot{\gamma}^{*}=10$, in agreement with previous results [6] where it has been shown that the critical shear rate depends on the chain length $N$. Note that the squared radius of gyration exhibits similar fluctuations and its variance could also be used to define the unfolding transition.

We next investigate the tension profile along the contour of a collapsed polymer subject to shear flow and present results for the tensile force $f_{i}$ as a function of bead position $i$ in fig. 2a for a few different shear rates. The polymer remains strongly collapsed for low shear rates $\dot{\gamma}<5$, as can be gathered from the small deviations in the radius of gyration, fig. 1a, and the small extensional fluctuations, fig. 1b. In such a globular conformation, as illustrated by the top snapshot in fig. 2e, tensile forces only stem from thermal fluctuations and are rather small and maximal at the termini. With increasing shear rate the maximal tensile force shifts away from the chain termini and a double-peak structure appears in the tensile force profile, as seen in fig. 2a, a transition taking place around $\dot{\gamma}=5$. We define the transition to be at the shear rate where the maxima move away from the chain termini. Notice that this is below the critical shear rate of unfolding, $\dot{\gamma}^{*}=10$, defined by the maximum variance of the exten- sion, fig. 1b. The double-peak structure is also observed for shear rates well above the unfolding transition, until the force profile changes around $\dot{\gamma}=30$ to a profile with a flat maximum in the middle and with strongly decreasing tension towards the termini. Fluctuations around the average forces $f_{i}$ are substantial, as indicated by the standard deviation defined by $\sigma_{i}^{2} \equiv \sigma^{2}\left(f_{i}\right)=\left\langle f_{i}^{2}\right\rangle-\left\langle f_{i}\right\rangle^{2}$ and shown for $N=50$ and different shear rates in fig. $2 \mathrm{~b}$. Corresponding broad normalized probability distributions $P\left(f_{25}\right)$ for the middle bead $i=25$ are shown in fig. 2c. Force profiles for different chain lengths are presented in fig. $2 \mathrm{~d}$ for a fixed shear rate $\dot{\gamma}=10$. We observe the double-peak structure only for long chains, $N \geq 20$. The tension for short chains at high shear are maximal in the middle and resemble inverted parabolas.

We attribute the peaks in the tensile force profiles close to the termini of the polymer to polymeric protrusions of length $l_{p}$, one of which is illustrated by the middle snapshot in fig. 2e. The reason for the existence of a force peak is that the hydrodynamic drag force acting on a protrusion is larger compared to beads within the globule, which is due to hydrodynamic shielding effects. In addition, the occurrence of the double-peak structure in the force profiles is closely related to the shear-induced unfolding transition that has been rationalized by a nucleation theory based on the presence of thermally excited polymeric protrusions [6]. The area shaded in gray in fig. 1 indicates the range $4<\dot{\gamma}<30$ where the double-peak structure in the tensile force profile appears. In the same range the variance of the squared extension $m\left(R_{S}^{2}\right)$ displays a maximum, fig. $1 \mathrm{~b}$, which is used to define the unfolding transition. In fact, the unfolding transition leads to cyclic elongation, illustrated by the bottom snapshot in fig. 2e, tumbling, and refolding, causing a large variance of the average extension. As the double-peak structure is only observed for long chains, $N \geq 20$, as seen in fig. 2 d, we conclude that chains have to be long enough so that a globule can form and the protrusion mechanism for unfolding becomes operational.

\subsection{Analysis}

In order to analyze the double-peak structure of the tensile force profiles figs. 2a,d in more detail, we define two characteristic quantities. First, the peak tensile force $f_{p}$, defined as the maximal average tension along the chain, which is associated with the average hydrodynamic drag force acting on protrusions. Second, the average protrusion length $l_{p}$, defined as the bead distance of the force peak from the adjacent chain end. The definition of the peak force $f_{p}$ and the protrusion length $l_{p}$ are illustrated by arrows in fig. $2 \mathrm{~d}$ for the right peak of the force profile for $N=50$. The length of a protrusion is also illustrated by the middle snapshot in fig. 2e. Since the tension profiles are symmetric with respect to the center of the polymer chain we consider the mean of the two peaks in order to determine $f_{p}$ and $l_{p}$. 
a)

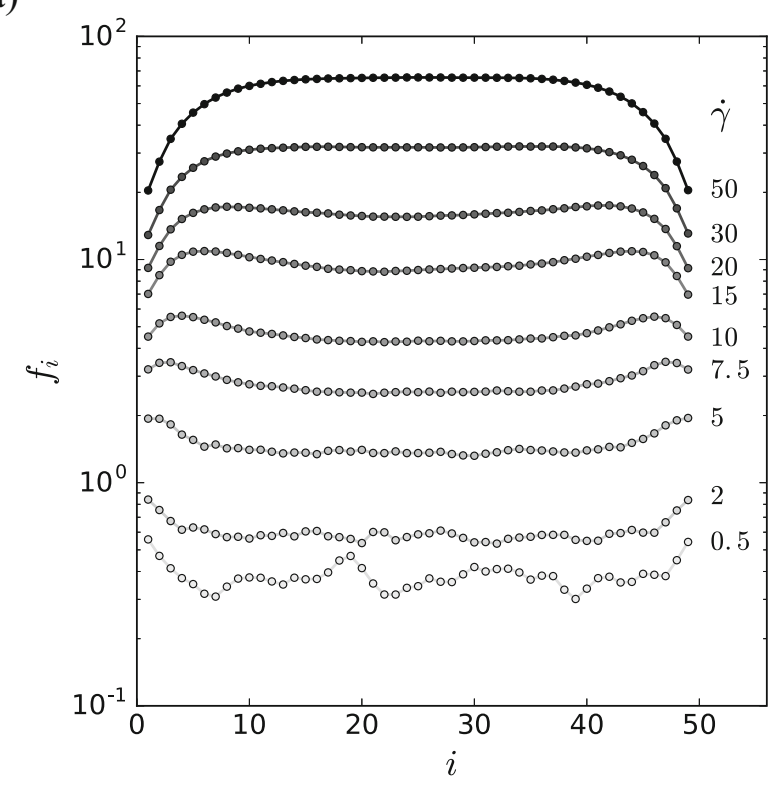

d)

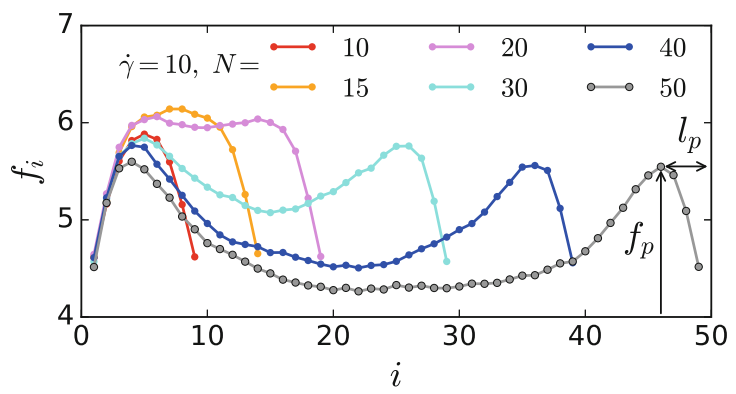

b)

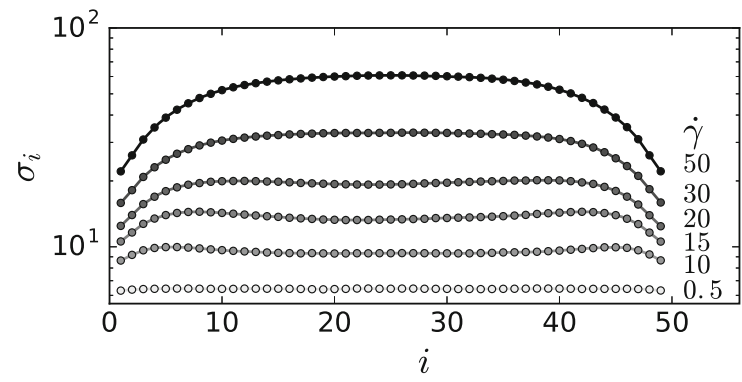

c)

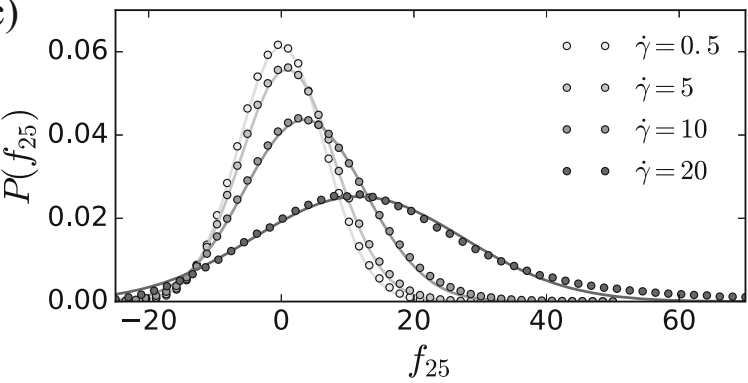

e)

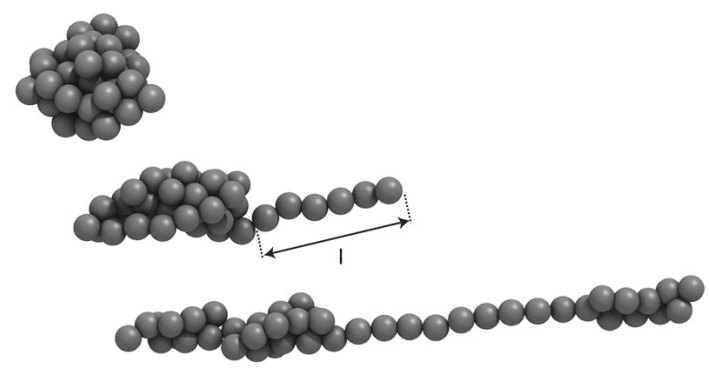

Fig. 2. a) Average tensile force $f_{i}$ as a function of bead position $i$ along a collapsed polymer with cohesive strength $\varepsilon=2$ and $N=50$. For increasing shear rate $\dot{\gamma}$ the overall tension increases and the profile changes at about $\dot{\gamma}=5$ from exhibiting maximal tensile forces at the termini to a double-peak structure, which disappears for larger $\dot{\gamma} \geq 30$. b) The standard deviation $\sigma\left(f_{i}\right)$ as a function of $i$ shown for different $\dot{\gamma}$ indicates large fluctuations. c) This is reflected in the broad normalized probability distribution $P\left(f_{25}\right)$ of the middle bead $i=25$ (symbols) that can be well fitted with a Gaussian (lines). d) Tension profiles for different chain length $N$ for fixed shear rate $\dot{\gamma}=10$. Black arrows illustrate the definition of the average protrusion length $l_{p}$ and the peak force $f_{p}$ for $N=50$. e) Snapshots of a collapsed globule, a globule having a protrusion of length $l_{p}$, and an elongated configuration (from top to bottom), all taken from simulations for $\varepsilon=2, N=50$ and $\dot{\gamma}=10$.

\subsubsection{Protrusion length and peak force at fixed cohesion and globule size}

In fig. 3 we present the average protrusion length $l_{p}$ and the average peak tensile force $f_{p}$ as a function of shear rate. As before, we consider a globule with cohesion $\varepsilon=2$ and $N=50$ beads. Hydrodynamic interactions (HI) are either taken into account (black filled symbols) or are neglected (FD, open symbols). In the FD case, no doublepeak structure is observed and with increasing shear rate the position $l_{p}$ of the peak force abruptly changes from the polymer termini to the middle. Note that this jump occurs around the critical shear rate of unfolding, which is at $\dot{\gamma}^{*}=1$ for the FD case [6]. For the HI case, the area shaded in gray in fig. 3 indicates the range $4<\dot{\gamma}<30$ where the double-peak structure appears. The protrusion length, fig. 3a, increases monotonically with shear rate according to the heuristic scaling law $l_{p} \sim \dot{\gamma}^{1.2}$ until the profile exhibits a single central peak at large shear rates. We observe protrusion lengths in the range $1<l_{p}<10$. Note that due to our discrete bead-spring model, we cannot determine arbitrarily short protrusions. When there is no protrusion and the maximal force is located at the chain termini, the smallest value is defined to be $l_{p}=1$. The data in fig. 3 is fitted only within the shaded area where double peaks are observed. The maximal forces, shown in fig. 3b, follow the scaling $f_{p} \sim \dot{\gamma}^{1.6}$ (black line) for large shear rates. For comparison we also plot (gray discs) the 
a)

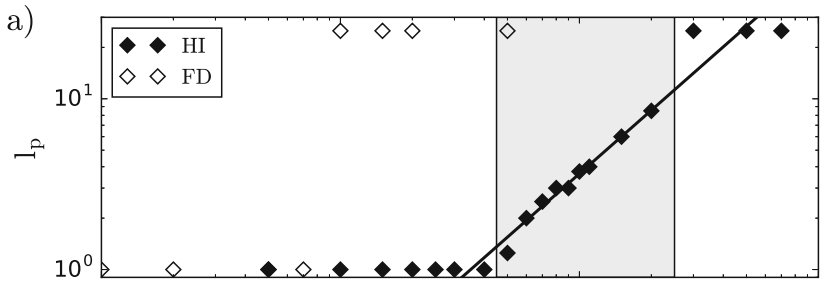

b)

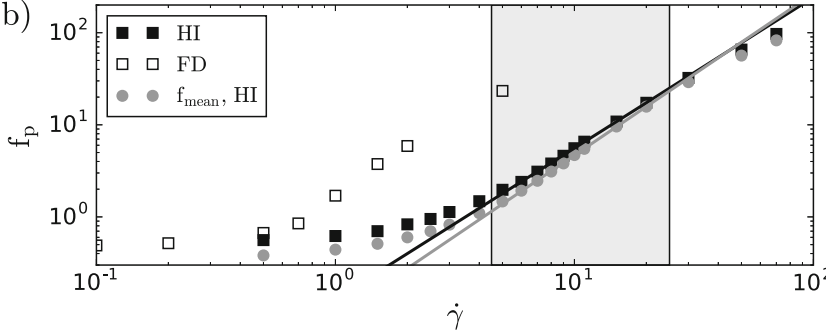

Fig. 3. A globular polymer with $N=50$ and $\varepsilon=2$ exhibits a double-peak structure only in the presence of hydrodynamic interactions (HI, filled symbols), the corresponding range of the shear rates $\dot{\gamma}$ is shaded in gray. a) The mean protrusion length $l_{p}$ as a function of $\dot{\gamma}$. In the free draining case (FD, open symbols) there is an abrupt jump around $\dot{\gamma}=1$ from the chain end to $l_{p}=25$ above which the maximal force is located in the middle of the chain. In the HI case the protrusion length increases according to $l_{p} \sim \dot{\gamma}^{1.2}$ (line) until there is only a single middle peak at large shear rates. b) The peak force $f_{p}$, defined as the maximum average tension along the polymer contour, scales as a function of shear rate like $f_{p} \sim \dot{\gamma}^{1.6}$, obtained by a fit of the HI data (black line) within the shaded area. Also the mean tension along the chain $f_{\text {mean }}$ (gray discs) is shown for the HI case which exhibits a similar scaling $f_{\text {mean }} \sim \dot{\gamma}^{1.8}$ (gray line).

mean tension along the chain, $f_{\text {mean }}=N^{-1} \sum_{i} f_{i}$, for varying shear rate, which shows a similar behavior as the peak force, $f_{\text {mean }} \sim \dot{\gamma}^{1.8}$ (gray line).

\subsubsection{Dependence on globule size and cohesive strength}

The hydrodynamic drag force acting on a protrusion could depend besides the protrusion length $l_{p}$ also on the globule radius $R \sim N^{1 / 3}$. In fact, the double-peak structure strongly depends on the chain length $N$, as indicated by the force profiles in fig. 2d. Another influential parameter is the cohesive strength $\varepsilon$ of the globule, which determines the restoring force on the protrusions. In the following we investigate the effect of different globule sizes and varying cohesive strength. We focus on the HI case, since no double-peak structure is observed in free draining simulations.

Results for the peak forces $f_{p}$ and the mean forces $f_{\text {mean }}$ as a function of $N$ are plotted in fig.4a for fixed cohesive strength $\varepsilon=2$ and shear rate $\dot{\gamma}=10$. The peak force only weakly depends on the globule size and decreases almost linearly with increasing chain length. The mean tensile force along the chain decreases more strongly with increasing $N$ and can be described by a power law $f_{\text {mean }} \sim N^{-1 / 3} \sim R^{-1}$, shown by the solid gray line.
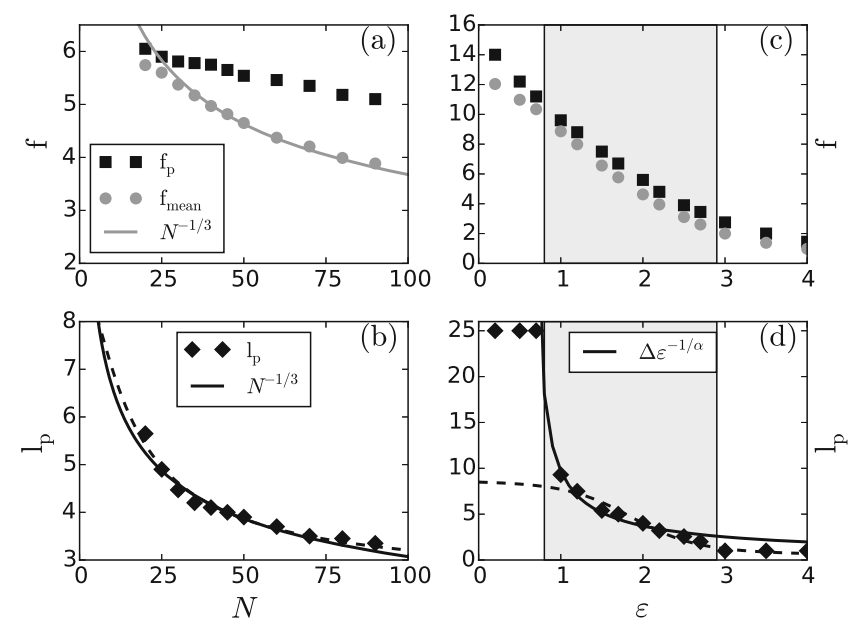

Fig. 4. a) Peak forces $f_{p}$, mean tension $f_{\text {mean }}$, and b) protrusion length $l_{p}$ as a function of globule size $N$ for fixed cohesive strength $\varepsilon=2$ and shear rate $\dot{\gamma}=10$. Solid lines indicate a scaling with the inverse radius of gyration $f, l_{p} \sim N^{-1 / 3} \sim R^{-1}$. The peak force in a) decreases only weakly and rather linearly with increasing globule size. The protrusion length in b) can be described in terms of a quasi-equilibrium theory by eq. (8) with fit parameters $\zeta_{\text {hyd }}=2.2 \times 10^{-4}, \zeta_{\text {coh }}=0.33$ (dashed line). c,d) Results for fixed globule size $N=50$ and shear rate $\dot{\gamma}=10$ at varying cohesive strength $\varepsilon$. A double-peak structure is only observed for $0.7<\varepsilon<3$ (shaded region) between the collapse transition at low cohesion and the freezing transition at high cohesion where no protrusions are observable. The tension in c) decreases approximately linearly with increasing $\varepsilon$. The dashed line in d) is the theoretical result eq. (8) with $\zeta_{\text {hyd }}=3.7 \times 10^{-4}, \zeta_{\text {coh }}=0.37$. Fitting the function eq. $(10)$ yields the exponent $\alpha=1.4$ (solid line).

The average protrusion length $l_{p}$, shown in fig. $4 \mathrm{~b}$, also decreases as the inverse of the radius $l_{p} \sim N^{-1 / 3} \sim R^{-1}$, shown by the solid black line.

The dependence on the cohesive strength $\varepsilon$ is shown in fig. 4c,d. A double-peak structure in the tension profile is only observed for values $0.7<\varepsilon<3$. The lower boundary is determined by the collapse transition that occurs for $N=50$ at $\varepsilon_{\text {col }} \approx 0.66$ [6], hence for lower values $\varepsilon<\varepsilon_{\text {col }}$ the polymer is in a coiled state where maximal forces occur in the chain middle. A higher cohesive strength $\varepsilon \approx 3$ leads to a compact, almost frozen globule which does not allow for protrusions. Both the peak force as well as the mean force decrease approximately linearly with increasing cohesive strength, as can be seen in fig. 4c. The protrusion length shown in fig. $4 \mathrm{~d}$ can be fitted within the shaded area by a power law $l_{p} \sim \Delta \varepsilon^{-1 / \alpha}$ (solid line), where we introduce the energy difference $\Delta \varepsilon=\varepsilon-\varepsilon_{\text {col }}$ and obtain the exponent $\alpha=1.4$. Note that this scaling behavior can be obtained by a quasi-equilibrium model and using the assumption that the average protrusion length is independent of the shear rate, as discussed in the next section. This approach yields an estimate for the exponent $\alpha$ that characterizes the restoring cohesive force on a protrusion defined via eq. (6). 


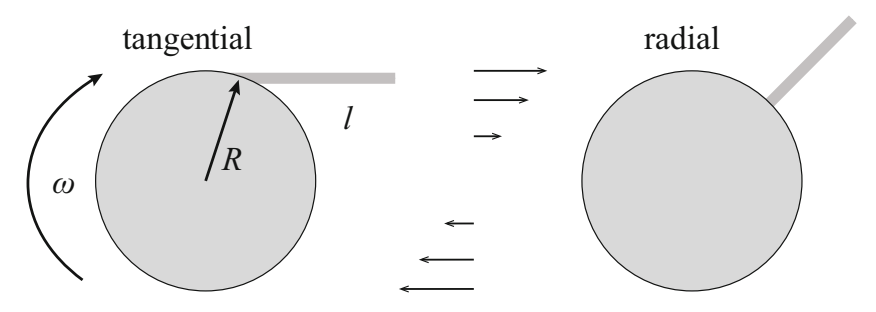

Fig. 5. Schematic illustration of a tangential and a radial protrusion of length $l$. The spherical globule with radius $R$ is rotating in shear flow with angular velocity $\omega=\dot{\gamma} / 2$.

\subsubsection{Quasi-equilibrium theory}

A previous study [6] established the scaling behavior for the hydrodynamic drag force and the restoring cohesive force on a protrusion in order to estimate the critical shear rate at which shear-induced globule unfolding sets in. It was argued that when the two opposing forces balance, there is an instability and the globule can fully elongate since no energy barrier prevents the protrusion to be dragged out from the globule. For a collapsed globule the unfolding time is presumably much larger than the inverse shear rate, i.e. the period of globule rotation. As a consequence, most protrusions are short-lived and are wrapped around the globule by the constant rotation rather than leading to full elongation of the polymer. Therefore a nucleation model is well suited to make scaling predictions for the transition from the collapsed to an unfolded state which is much slower compared to the fast shear-induced refolding. This might be the reason why protrusions are absent in the FD case, where hydrodynamic shear freely penetrates the globule and little resistance against shearinduced deformations leads to fast unfolding at relatively low shear rates.

In contrast to these nucleation-type arguments, here we make use of the scaling behavior of drag force and cohesive force and apply a quasi-equilibrium theory in order to estimate average values for the protrusion length $l_{p}$ and the tensile force $f_{p}$. Our quasi-equilibrium theory does not describe the rare events of full unfolding around the critical shear rate but yields information about the average protrusions which can be compared to our tensile force profiles. As schematically depicted in fig. 5, we consider a polymer segment of length $l$ protruding from a collapsed spherical globule with radius $R$, which is rotating in shear flow with angular velocity $\omega=\dot{\gamma} / 2$. In principle we would have to take into account all possible configurations of protrusions, however, we focus on the cases where the largest drag force occurs. Two typical cases are shown in fig. 5: for a tangential protrusion the drag force is maximal at the top of the globule (left), while the force on a radial protrusion is maximal at an angular position of $\pi / 4$ with respect to the flow direction (right) [6].

In the presence of hydrodynamic interactions, the drag force results from an integral along the protrusion contour, $f_{\text {hyd }}=\int_{0}^{l}\left(\mathbf{v}-\mathbf{v}_{0}\right) \mathrm{d} \mathbf{l}$, for this we use the analytical flow profile around a sphere in linear shear [33],

$$
v_{\beta}=\dot{\gamma} z \delta_{\beta x}+\frac{\dot{\gamma} R}{2}\left(\frac{5\left(1-(r / R)^{2}\right) \beta x z}{R^{3}(r / R)^{7}}-\frac{\delta_{\beta z} x+\delta_{\beta x} z}{R(r / R)^{5}}\right),
$$

with $\beta \in\{x, y, z\}$. We define the reference velocity $\mathbf{v}_{0}$ at the surface of the globule from where the protrusion emerges. Expansion for short length $l<R$ of the velocity profile eq. (4) leads to a radial velocity $v \sim \dot{\gamma} l^{2} / R[6]$ and hence to

$$
f_{\text {hyd }} \sim \dot{\gamma} l^{3} / R
$$

Using the tangential configuration of the protrusion only changes the prefactor but leaves the scaling behavior unchanged.

The restoring cohesive force is assumed to scale as

$$
f_{\text {coh }} \sim-\Delta \varepsilon l^{\alpha-1},
$$

where $\Delta \varepsilon=\varepsilon-\varepsilon_{\text {col }}$ is the cohesive strength relative to the cohesion at the collapse transition. The linear behavior of the force with respect to the cohesive strength $\Delta \varepsilon$ is indeed reflected in our simulation data, as shown in fig. 4c. The parameter value $\alpha=1$ describes protrusions much longer than the globule-solvent interfacial width and which experience a constant cohesive force that does not depend on the length $l$. For short protrusions the harmonic approximation is expected, $\alpha=2[6]$.

In order to calculate the average protrusion length $l_{p}$, we construct the energy expression for a protrusion

$$
U_{\text {tot }}=U_{\text {hyd }}+U_{\text {coh }}=-\zeta_{\text {hyd }} \dot{\gamma} l^{4} / R+\zeta_{\mathrm{coh}} \Delta \epsilon l^{\alpha},
$$

where the potential of mean force associated with the cohesion is determined by $\partial U_{\text {coh }} / \partial l=-f_{\text {coh }}$ and the contribution that pulls the protrusion out of the globule is given by $\partial U_{\text {hyd }} / \partial l=-f_{\text {hyd }}$. The prefactors $\zeta_{\text {hyd }}$ and $\zeta_{\text {coh }}$ are used to fit the data. We next assume that the protrusion configuration relaxes relatively quickly and can be described by a quasi-equilibrium Boltzmann distribution. Numerical evaluation of the Boltzmann average yields the average protrusion length

$$
l_{p}=\frac{\int_{0}^{l_{\max }} e^{-U_{\mathrm{tot}}(l)} l \mathrm{~d} l}{\int_{0}^{l_{\max }} e^{-U_{\mathrm{tot}}(l)} \mathrm{d} l},
$$

where the integration boundary $l_{\max }$, the maximal protrusion length, is a further parameter that is used to fit the simulation data. Similarly, an estimate for the average peak force is given by

$$
f_{p}=\frac{\int_{0}^{l_{\max }} e^{-U_{\mathrm{tot}}(l)}\left|\frac{\partial U_{\mathrm{tot}}}{\partial l}\right| \mathrm{d} l}{\int_{0}^{l_{\max }} e^{-U_{\mathrm{tot}}(l)} \mathrm{d} l} .
$$

In the limit of vanishing shear rate or large globules, or under the assumption that the average protrusion length is independent of the shear rate, we can neglect the first term in eq. (7) and calculate the average protrusion length via eq. (8) for $l_{\max } \rightarrow \infty$, which yields

$$
l_{p} \sim \Delta \varepsilon^{-1 / \alpha} .
$$




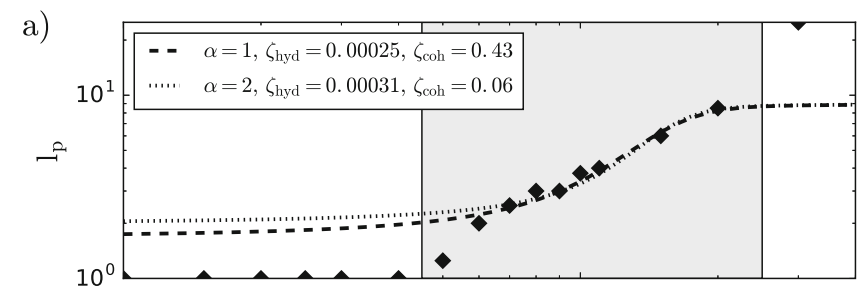

b)

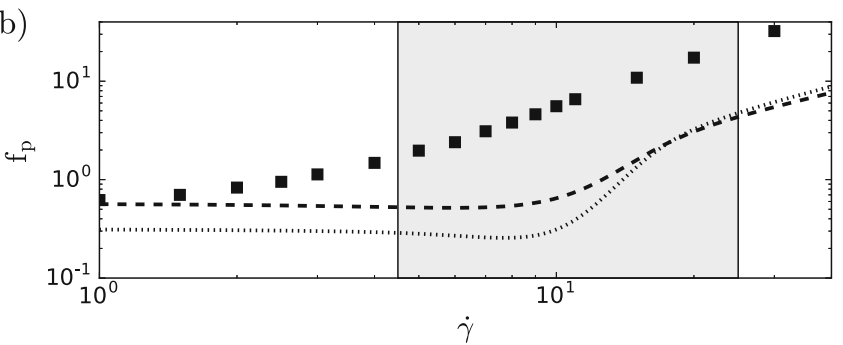

Fig. 6. a) Average protrusion length $l_{p}$ and b) peak force $f_{p}$ as a function of shear rate for simulations with HI, $N=50$ and $\varepsilon=2$. Lines in a) represent theoretical results eq. (8) with exponent $\alpha=1$ (dashed line), $\alpha=2$ (dotted line) and fitting parameters $\zeta_{\text {hyd }}$ and $\zeta_{\text {coh }}$ (values in the legend). Lines in b) correspond to eq. (9) using the same parameters as in a). The shaded area indicates where double peaks in the tension profile are observed.

The dashed lines in fig. $4 \mathrm{~b}$ and fig. $4 \mathrm{~d}$ represent the result eq. (8) for the protrusion length as function of chain length $N$ and as a function of the cohesive strength $\varepsilon$, respectively. We use the known parameter values $R=R_{g}^{0}=$ $\sqrt{11.3}$ for $N=50, \Delta \varepsilon=\varepsilon-\varepsilon_{\mathrm{col}}=1.34$ for $\varepsilon=2$, and assume long protrusions characterized by $\alpha=1$, $l_{\max }=9$, which is an estimate deduced from fig. 3a. Note that we neglect the dependence of the collapse transition on the chain length and use a constant $\varepsilon_{\text {col }}$. Satisfactory agreement is obtained between the theoretical result and the simulation data by adjusting the fit parameters $\zeta_{\text {hyd }}=2.2 \times 10^{-4}$ and $\zeta_{\text {coh }}=0.33$ for fig. $4 \mathrm{~b}$ and $\zeta_{\text {hyd }}=3.7 \times 10^{-4}$ and $\zeta_{\text {coh }}=0.37$ for fig. $4 \mathrm{~d}$. The differences in the fitting factors might be due to the simplifying assumption that the maximal protrusion length $l_{\text {max }}$ as well as $\varepsilon_{\text {col }}$ are kept constant.

We also compare the prediction of the quasi-equilibrium theory for the average protrusion length $l_{p}$, eq. (8), as a function of shear rate in fig. 6 a for the same data as shown in fig. 3a. The result for $\alpha=1$ is shown as a dashed curve and for $\alpha=2$ as a dotted line. It can be seen that the simple theory does not describe the simulation data very accurately in the transition region. In fig. $6 \mathrm{~b}$ we compare the peak force measured in simulations with the theoretical prediction, eq. (9), using the same fit parameters $\zeta_{\text {hyd }}$ and $\zeta_{\text {coh }}$ as obtained from fig. 6 a. Since the analytical prediction largely underestimates the simulation data we conclude that the simple combination of Boltzmann averaged length $l_{p}$ and peak force $f_{p}$ does not yield a consistent description of average protrusions. A somewhat better result is obtained for a small value $\alpha=1$, which is in contradiction to the result of ref. [6] where the scaling behavior of the critical shear rate suggests $\alpha=2$ for the HI case and hence short protrusions. We conclude that the quasi-equilibrium theory would have to be extended in order to consistently capture all properties of shear-induced protrusions.

An interesting question concerns the actual value of the exponent $\alpha$ characterizing the cohesive force on a protrusion defined in eq. (6). The scaling relation eq. (10) shown for fixed shear rate and globule size in fig. $4 \mathrm{~d}$ as a solid line was not only introduced as a phenomenological power law, but it is also obtained when calculating the average protrusion length eq. (8) and neglecting the first term of eq. (7). Despite this simplistic assumption (note that both shear rate, shown in fig. 3a, and globule size, shown in fig. $4 \mathrm{~b}$, influence the average protrusion length) we can estimate the parameter $\alpha=1.4$. This corresponds to an intermediate value between long protrusions and constant cohesive force, $\alpha=1$, and short protrusions, $\alpha=2$.

In conclusion, the quasi-equilibrium theory contains sufficient fit parameters in order to roughly match simulations results for the average protrusion length. This might be surprising as the time scale governing the transition between collapsed and unfolded state is large compared to the shortest relevant time scale in the system, which is the period of globule rotation, and thus rare events of full polymer elongations are presumably not accurately described by our quasi-equilibrium model. In line with this, the Boltzmann averaging of both $l_{p}$ and $f_{p}$ with the same fit parameters does not yield a consistent description of the average protrusion force measured in the simulation. In order to improve the accuracy of our model we used the full velocity profile, eq. (4), for the calculation of the energy term $U_{\text {hyd }}$ associated with the hydrodynamic drag on the protrusion instead of the approximation for short protrusions in eq. (5), but found no qualitative difference, only the prefactor $\zeta_{\text {hyd }}$ changes (data not shown). A further possible improvement of the theory might be to average over all possible protrusion configurations or to consider a maximal cut-off length $l_{\max }$ that depends on globule size, cohesive strength, and shear rate.

\section{Connection between tension profile and VWF proteolysis}

The tension profiles that we investigated in the previous section can help to explain the shear-induced activation of collapsed biopolymers from a fundamental polymer physics point of view. In particular, in this section we make the connection between the shear-induced tension along a polymer chain and the shear-dependent proteolytic degradation of the multimeric blood protein von Willebrand factor (VWF) by its specific cleavage enzyme ADAMTS13. The ADAMTS13-mediated cleavage of fulllength VWF in shear has been measured recently [30] using fluorescence correlation spectroscopy (FCS) in combination with a microfluidic shear cell. The effect of shear on the kinetics of VWF cleavage in aqueous buffer and in blood plasma was quantified by measuring the timeresolved increase in molar VWF multimer concentration. 
One of the main results of that study was a strong sigmoidal increase of ADAMTS13 activity in plasma as a function of shear rate.

As has been shown previously, external forces induce an opening of the VWF A2 domain which thereby becomes accessible for cleavage by ADAMTS13 [14,28]. In order to describe the opening process of the A2 domain required for cleavage by ADAMTS13, we employ a simple two-state model where a single subunit is either closed or open. The kinetics is described by the force-dependent opening and closing rates that determine the probability for the cleavage site to be in the open state, which in turn can be related to the shear-dependent cleavage rate measured in the experiment [30]. This allows to deduce parameters characterizing the process of stochastic domain opening and closing such as an effective force scale and the free energy difference from experimental shear-dependent measurements.

\subsection{Morrison kinetics without shear flow}

In the absence of shear flow, previous experiments measured the ADAMTS13-mediated cleavage of VWF under denaturing buffer conditions [30]. In order to observe a finite cleavage activity without shear flow, the denaturant is essential to render cleavage sites accessible. The results are shown in fig. 7, where the cleavage rate increases with VWF multimer concentration $C_{V}$ depending on the enzyme concentration $C_{A}$. In the context of enzyme kinetics, the cleavage rate can be described by the Morrison equation [34],

$$
\begin{aligned}
k_{\mathrm{CR}}= & \frac{\mathrm{d} C_{V}}{\mathrm{~d} t}=\frac{\tilde{k}_{\mathrm{cat}}}{2}\left(K_{M}+C_{A}+\bar{N}_{\text {open }} C_{V}\right. \\
& \left.-\sqrt{\left(K_{M}+C_{A}+\bar{N}_{\text {open }} C_{V}\right)^{2}-4 C_{A} \bar{N}_{\text {open }} C_{V}}\right)
\end{aligned}
$$

with the catalytic rate constant $\tilde{k}_{\text {cat }}$ and the MichaelisMenten constant $K_{M}$. The central assumption is that the cleavage rate depends on the mean number of accessible cleavage sites per VWF multimer, $\bar{N}_{\text {open }}$, via the effective substrate concentration $\bar{N}_{\text {open }} C_{V}$. Equation (11) is a generalized Michaelis-Menten equation without the free ligand approximation, i.e., it is valid for $K_{M}$ values smaller than $C_{A}$ and does not assume the free substrate concentration to be equal to the total substrate concentration, an approximation that might not be valid when accessible cleavage sites are sparse. Solid lines in fig. 7 represent global fits of the experimental data to eq. (11) with parameters $\tilde{k}_{\text {cat }}=0.001 \mathrm{~s}^{-1}, K_{M}=2.3 \mathrm{nM}$, and the number of accessible cleavage sites $\bar{N}_{\text {open }}=0.04$. This means that the denaturing buffer only opens a small fraction of the cleavage sites per multimer. Our results suggest domain opening to be the cleavage rate limiting factor rather than the ADAMTS13 concentration, since the $K_{M}$ is below the physiological value of the ADAMTS13 concentration, which is about $C_{A} \approx 5 \mathrm{nM}[30]$.

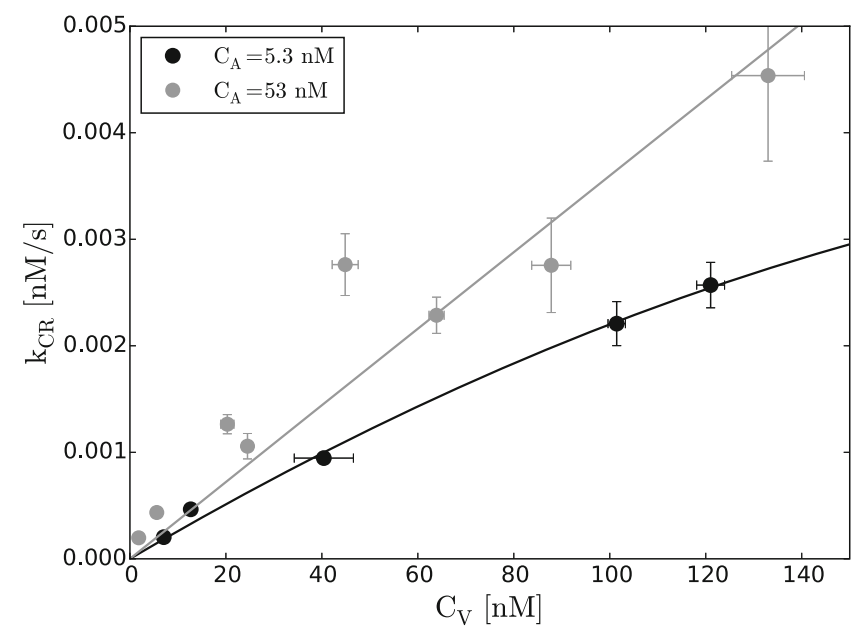

Fig. 7. Experimental data from [30] for the cleavage rate in denaturing buffer as a function of VWF multimer concentration $C_{V}$ for two different ADAMTS13 concentrations $C_{A}=5.3 \mathrm{nM}$ (black) and $C_{A}=53 \mathrm{nM}$ (grey). Lines illustrate Morrison kinetics eq. (11) with fit parameters $\tilde{k}_{\text {cat }}=0.001 \mathrm{~s}^{-1}$, $K_{M}=2.3 \mathrm{nM}$, and $\bar{N}_{\text {open }}=0.04$.

The Michaelis-Menten constant of $K_{M}=962 \mathrm{nM}$ determined previously [30] results from the alternative assumption that all cleavage sites are accessible under denaturing conditions, i.e. $\bar{N}_{\text {open }}=N_{m}$, where the average number of monomers is obtained by the experimentally determined VWF length distribution [25]

$$
N_{m}=2 \frac{\sum_{N=1}^{\infty} N n^{N-1}}{\sum_{N} n^{N-1}}=5.6,
$$

with the parameter $n=0.64$. Note that there are two cleavage sites per dimer (bead). Here, we present the alternative approach of introducing the mean number of accessible cleavage sites $\bar{N}_{\text {open }}$ as a fit parameter, which allows the consistent description of shear-dependent experiments, as explained in sect. 4.3. The simultaneous fit of the two different enzyme concentrations in fig. 7 with the same $\tilde{k}_{\text {cat }}=0.001 \mathrm{~s}^{-1}$ is an advantage compared to the method used previously [30], where the catalytic rate constant depends on $C_{A}$. More experimental data for different enzyme concentrations would be needed in order to consolidate the present fitting approach.

\subsection{Model for stochastic cleavage site opening under tensile forces}

In our homopolymer model, a spherical bead of radius $a$ represents the VWF's repeating unit, i.e. a dimer. Since rescaled shear rates $\dot{\gamma}=\tilde{\dot{\gamma}} \tau$ are used with a timescale $\tau=6 \pi \eta a^{3} / \mathrm{kT}$, simulation results can be interpreted in terms of arbitrary values of radius and viscosity. In order to compare the dimensionless simulation values to physical units, we use the viscosity $\eta=1.2 \times 10^{-3} \mathrm{Pas}$, and temperature $T=310 \mathrm{~K}$. The remaining parameters are the 
bead radius $a$ and the cohesive strength $\varepsilon$, which strongly influence the critical shear rate at which shear-induced unfolding of the polymeric globule sets in [6]. We choose the cohesive parameter $\varepsilon=2$ so that in the absence of shear flow the polymer is collapsed but still far from the freezing transition at around $\varepsilon_{\text {freeze }}=4$ [35]. The dimensionless critical shear rate that we determine for a globule with $N=50$ is about $\dot{\gamma}^{*}=10$ (fig. 1b), which translates to the experimental value $\tilde{\dot{\gamma}}_{\text {exp }}^{*} \approx 5000 \mathrm{~s}^{-1}$ [9] when using the bead radius $a=73 \mathrm{~nm}$. This compares well with literature values of the VWF dimer size ranging from $60 \mathrm{~nm}$ to $82 \mathrm{~nm}$ [36-38]. Note that the critical shear rate also depends on the bead number [6], however, here for simplicity we consider a system with $N=50$ beads.

From simulations we obtain the average tension $f_{i}$ between adjacent beads (figs. 2a,d) and calculate the probability for a cleavage site to be open, given by

$$
P_{i}=\frac{1}{1+e^{\Delta F} e^{-f_{i} / f_{e}}} .
$$

This expression can be derived by considering the reversible reaction of the cleavage site from being in the closed state to the open state, which we assume to be separated by a transition state energy barrier. The corresponding rate equation for the time-dependent probability to be in the open state $P_{i}$ is given by

$$
\mathrm{d} P_{i} / \mathrm{d} t=k_{o}\left(1-P_{i}\right)-k_{c} P_{i} .
$$

Whereas the opening rate $k_{o}=k_{o}^{0} \exp \left(f_{i} / f_{o}\right)$ increases exponentially with tension according to the characteristic opening force scale $f_{o}$, the closing rate $k_{c}=$ $k_{c}^{0} \exp \left(-f_{i} / f_{c}\right)$ is assumed to decrease with tension according to the closing force scale $f_{c}$ (which in general is different from $f_{o}$ ). Opening and closing rates in the absence of force are defined by $k_{o}^{0}$ and $k_{c}^{0}$. In a stationary state, the time derivative of $P_{i}$ vanishes and we obtain eq. (13), where the effective force scale is defined by

$$
f_{e}=1 /\left(1 / f_{o}+1 / f_{c}\right)
$$

and the unstressed equilibrium constant $k_{o}^{0} / k_{c}^{0}=\exp$ $(\Delta F)$ defines the free energy difference $\Delta F$ between open and closed state. Compared to the length $x_{o}=\mathrm{kT} / f_{o}$, which is the distance along the reaction coordinate from the closed state to the transition state, the distance $x_{c}=$ $\mathrm{kT} / f_{c}$ between transition state and fully open state, e.g. the unfolded A2 domain stretched to its contour length, is presumably much larger and thus $f_{c} \ll f_{o}$. Consequently, from eq. (15) we conclude that the force scale of the closing transition dominates the effective force scale $f_{e} \approx f_{c} \ll f_{o}$.

Considering the entire polymer, we define the mean number of accessible cleavages sites as

$$
N_{\text {open }}(N)=2 \sum_{i}^{N} P_{i},
$$

where the prefactor accounts for the fact that every dimer exhibits two cleavage sites. The cleavage process consists of three steps: the opening of the cleavage site, diffusion of the enzyme to this active site, and the actual catalytic cleavage. Our underlying assumption is that the domain opening and not diffusion or the chemical reaction is the rate limiting mechanism for the cleavage process. We thus assume that when there is an open cleavage site, the probability being described by eq. (13), the diffusion rate as well as the reaction rate are large enough such that VWF is readily cleaved.

In order to obtain the size-averaged number of open cleavage sites, the weighted arithmetic mean is calculated

$$
\bar{N}_{\text {open }}=\frac{\sum_{N}^{N_{\max }} n^{N-1} N_{\text {open }}(N)}{\sum_{N}^{N_{\max }} n^{N-1}}
$$

according to an exponential VWF size distribution with the parameter $n=0.64$, which was determined by fluorescence correlation spectroscopy of VWF in blood plasma [25]. Simulation results are used to calculate $N_{\text {open }}(N)$ and the upper boundary in the sums of eq. (17) is set to a maximal chain length $N_{\max }=10$. Taking into account longer chains does not alter the result due to the negligible weights for large $N$ in eq. (17).

\subsection{Mapping simulation results and experiments of shear-induced VWF cleavage}

Experimental cleavage rates of VWF in blood plasma [30] as a function of shear rate, shown in fig. 8a as black symbols, exhibit a steep increase corroborating the concept of shear-induced opening of the A2 cleavage domain.

The experimental data can be described by a phenomenological sigmoidal function

$$
k_{\mathrm{CR}}=k_{\max } \frac{1}{1+e^{-\left(\tilde{\gamma}-\tilde{\gamma}_{c}\right) / \Delta \tilde{\gamma}}},
$$

illustrated as a black line in fig 8a, with fitting parameters $\Delta \tilde{\dot{\gamma}}=1271 \mathrm{~s}^{-1}$ and $\tilde{\dot{\gamma}}_{c}=5522 \mathrm{~s}^{-1}$, the latter being interpreted as the half maximum shear rate. The enzyme activity dependent prefactor $k_{\max }=0.0035 \mathrm{nM} / \mathrm{s}$ denotes the maximal enzymatic rate in the case of fully accessible cleavage sites. Notice that high cleavage activity is closely related to the shear-induced VWF unfolding transition since the half maximum shear rate $\tilde{\dot{\gamma}}_{c}$ agrees with the critical shear rate of unfolding, $\tilde{\dot{\gamma}}_{\text {exp }}^{*} \approx 5000 \mathrm{~s}^{-1}[9]$, where the polymer size fluctuations are maximal.

The connection to simulation results is established using the Morrison eq. (11) in combination with the shear-dependent mean number of accessible cleavage sites, eq. (17) with eqs. (13) and (16). The tensile force profiles $f_{i}$ are taken from our simulations in the previous sect. 3 and we use the Michaelis-Menten constant $K_{M}=2.3 \mathrm{nM}$ determined in sect. 4.1 under denaturing buffer conditions since we expect that value not to be very different in blood plasma. The ADAMTS13 concentration in the blood plasma was $C_{A}=1.3 \mathrm{nM}$ and the VWF concentration $C_{V}=13 \mathrm{nM}[30]$. As a result of a least square fit, as seen in fig. 8a, simulations results (cyan symbols) match 
a)

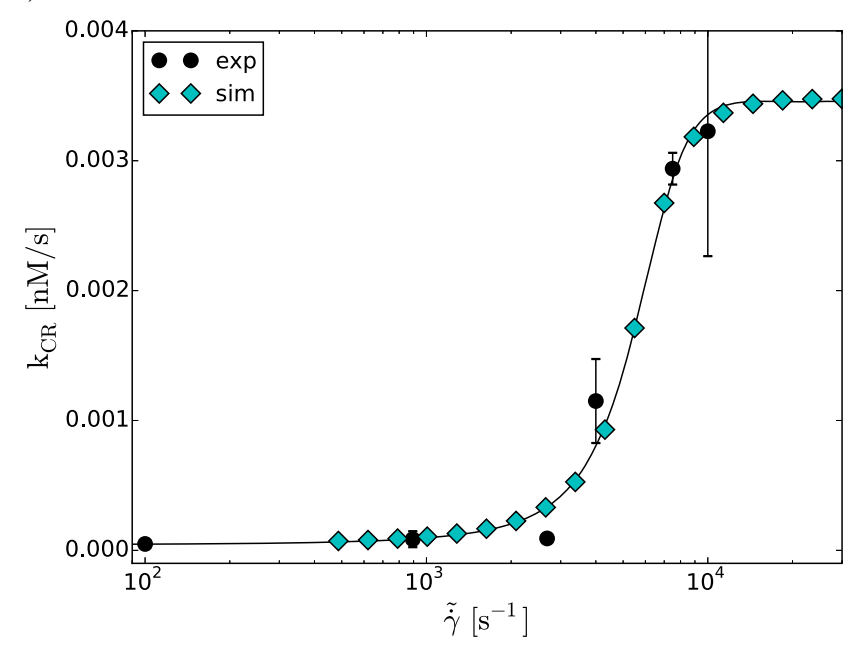

b)

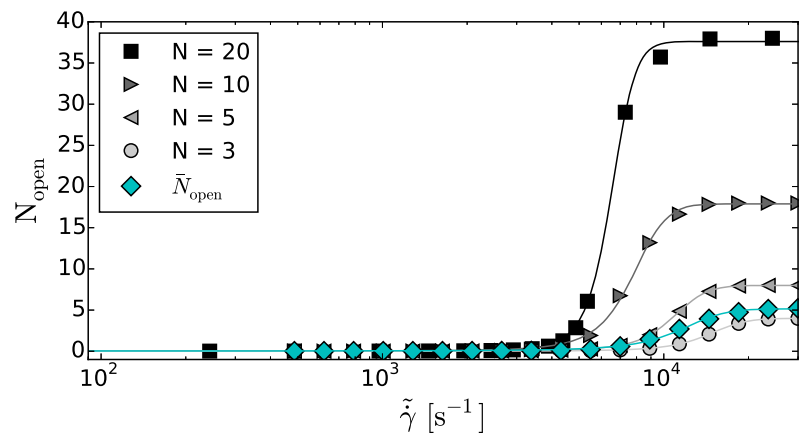

Fig. 8. a) Experimental data for the cleavage rate of VWF in blood plasma (black symbols, data from [30]) as a function of shear rate is described by the phenomenological sigmoidal function, eq. (18), as a solid line. Assuming a stochastic model for the description of the probability of a subunit to be open, eq. (13), and the mean number of open cleavage sites $\bar{N}_{\text {open }}$ to determine the substrate concentration in the Morrison eq. (11), agreement with simulation data (cyan symbols) is obtained for fitting parameter values $\Delta \tilde{F}=8.5 \mathrm{kT}, \tilde{f}_{e}=0.06 \mathrm{pN}$, and a catalytic rate constant $\tilde{k}_{\text {cat }}=0.0027 \mathrm{~s}^{-1}$. b) Simulation results for the mean number of open cleavage sites, eq. (16), as a function of shear rate for a few different chain lengths $N$. The size-averaged number $\bar{N}_{\text {open }}$ according to eq. (17) is denoted by cyan symbols. Lines are fits to a sigmoidal function according to eq. (18)

the sigmoidal description (solid line) of the experimental data. We obtain the fit parameters $\Delta F=8.5$ in units of $\mathrm{kT}$ and $f_{e}=1.0$ in rescaled units characterizing the probability of single cleavage sites to be accessible, eq. (13), as well as the catalytic rate constant $\tilde{k}_{\text {cat }}=0.0027 \mathrm{~s}^{-1}$, which is in satisfactory agreement with the $0.001 \mathrm{~s}^{-1}$ obtained in the absence of shear flow, fig. 7. The force scale in physical units is given by $\tilde{f}_{e}=f_{e} \mathrm{kT} / a=0.06 \mathrm{pN}$. Notice that the length scale $\tilde{x}_{c} \approx \tilde{x}_{e}=\mathrm{kT} / \tilde{f}_{e}=69 \mathrm{~nm}$ associated with the distance along the reaction coordinate between the open state and the transition state is comparable to the contour length of the A2 domain of $58 \mathrm{~nm}$ [13].
The dependence of the mean number of accessible cleavage sites on the chain length $N$ is illustrated in fig. $8 \mathrm{~b}$. For a few different lengths we plot $N_{\text {open }}$ as a function of the shear rate, using the parameters obtained before: $\Delta F=8.5$ and $f_{e}=1.0$. Lines represent sigmoidal fits according to eq. (18). The saturating value is proportional to the monomer number and with increasing $N$ the transition shifts towards lower values of the shear rate indicating that the probability to find open cleavage sites is higher for longer polymers. We also plot the size-averaged number according to eq. (17) denoted by cyan symbols.

\subsection{Alternative models for mean number of accessible cleavage sites}

\subsubsection{Alternative model I}

In this section, we present an alternative definition of the mean number of open cleavage sites $\bar{N}_{\text {open }}$, eq. (17), that determines the shear-dependence of the Morrison eq. (11). We employ an analytical approach to calculate the probability of cleavage sites to be open based on our scaling results from sect. 3.1.1. While the model eq. (13) includes simulation results directly via the measured average forces, $f_{i}$, along the polymer chains, here we consider the mean force, $f_{i} \approx f_{\text {mean }}$, which depends on the shear rate according to $f_{\text {mean }} \sim \dot{\gamma}^{1.8}$, as seen in fig. $3 \mathrm{~b}$. We thus assume equal probability for any cleavage site to be open and obtain for the mean number of open cleavage sites

$$
\bar{N}_{\text {open }}=N_{m} \frac{1}{1+e^{\Delta F} e^{-\left(\tilde{\gamma} / \tilde{\gamma}_{e}\right)^{1.8}}},
$$

where the average number of monomers is given by eq. (12). Following the same fitting procedure as before, we obtain the fit parameters $\Delta F=4.9, \tilde{\hat{\gamma}}_{e}=3322 \mathrm{~s}^{-1}$, and $\tilde{k}_{\text {cat }}=0.0028 \mathrm{~s}^{-1}$ and plot the result in fig. 9 as a red dashed line. The analytical approach using a power law for the shear-dependence of the mean tensile forces yields a suitable description of the experimentally observed cleavage rate. Compared to the result from sect. 4.3, the catalytic rates agree very well but the free energy difference is smaller by $3.6 \mathrm{kT}$. We conclude that based on the quality of fits in fig. 9 it is not possible to decide whether the assumption underlying eq. (17) or eq. (19) is valid. This means that it remains unclear to what extent the inhomogeneous force profiles as shown in fig. 2 influence the cleavage process of VWF multimers. It therefore might be possible that cleavage occurs predominantly at the sites of maximal tensile forces along the multimer contour.

\subsubsection{Alternative model II}

Another method to model the fraction of accessible cleavage sites and thereby the cleavage rate is based on the observation of broad distributions of the tensile forces, shown in fig. 2c. These are well fitted by a Gaussian,

$$
G_{i}(f)=\exp \left(-\left(f-f_{i}\right)^{2} /\left(2 \sigma_{i}^{2}\right)\right) / \sqrt{2 \pi \sigma_{i}^{2}} .
$$




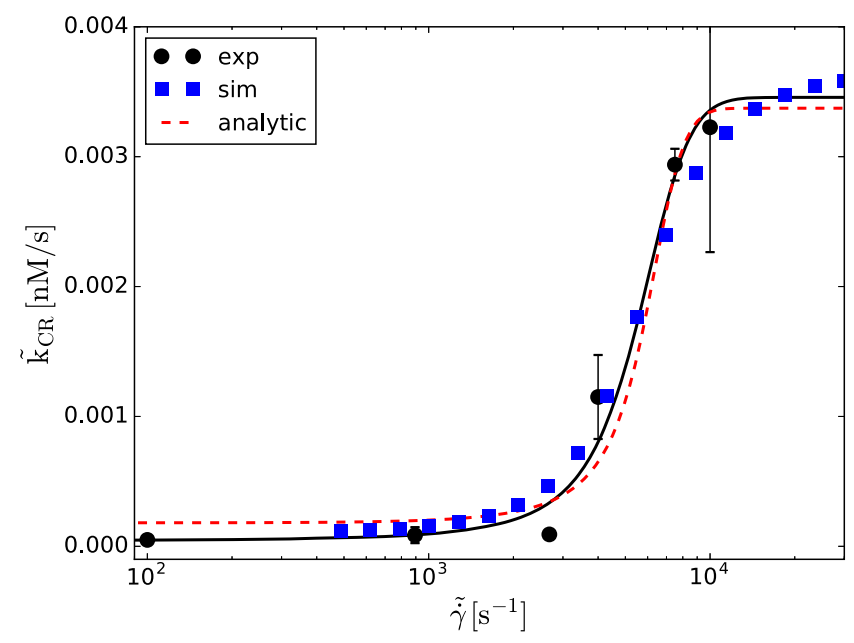

Fig. 9. Alternative models describing experimental cleavage rates in blood plasma (black symbols) as a function of shear rate. The black solid line represents the phenomenological sigmoidal function, eq. (18). The analytical model using the mean number of open cleavage sites, eq. (19), and the fit of eq. (11) (red dashed line) yields a very similar result for the cleavage rate. Instead of using the average forces $f_{i}$ for the calculation of the probability $P_{i}$, eq. (13), for the blue symbols we use the average of $P_{i}$, eq. (21), which takes into account the full distribution of tensile forces.

The resulting mean force $f_{i}$ and standard deviation $\sigma_{i}$ can be used to calculate the average probability of the cleavage site to be open

$$
\hat{P}_{i}=\int_{-\infty}^{\infty} G_{i}(f) \frac{1}{1+e^{\Delta F} e^{-f / f_{e}}} \mathrm{~d} f .
$$

In contrast to eq. (13), where only the average forces $f_{i}$ are used to calculate the probability of an open cleavage site, eq. (21) takes into account the full distribution of tensile forces. The result of the fit of eq. (11) using $N_{\text {open }}(N)=2 \sum_{i}^{N} \hat{P}_{i}$ and eq. (17) is shown in fig. 9 by blue squares with fit parameters $\Delta F=12.5, f_{e}=1.67$ and $\tilde{k}_{\text {cat }}=0.0029 \mathrm{~s}^{-1}$. Although this method leads to an adequate graphical description of the cleavage rate, the resulting fit parameters should be interpreted with care since a wide range of values for $\Delta F$ and $f_{e}$ lead to fits of comparable quality. The large free energy difference $\Delta F=12.5$ between the open and the closed state might suggest that when using the full tension distribution one overestimates the influence of the force on the probability to find open cleavage sites. In conclusion, it remains unclear to what extent the strong fluctuations of the tensile force, which are due to the rare events of full polymer unfolding, contribute to the cleavage domain opening that determines the shear-induced cleavage process.

\subsection{Comparison to VWF A2 domain unfolding upon external stretching force}

In the following we make the connection to the forceinduced unfolding of the isolated A2 domain measured with optical tweezers [13], where it is hypothesized that unfolding of A2 is required for cleavage by ADAMTS13. In that study, unfolding forces are measured as a function of force loading rates. In line with our assumption, the unfolding rate $k_{u}=k_{u}^{0} \exp \left(f / f_{u}\right)$ is assumed to increase exponentially with applied force $f$, and the characteristic force scale $f_{u}$ of $\mathrm{A} 2$ unfolding is determined. Note that the unfolding of A2 probed in the force spectroscopy experiments is not necessarily the same process as probed in the ADAMTS13 cleavage experiments in shear, as we will explain in the following.

In our analysis in sect. 4.2 we identify the closing force scale $f_{c}$ to be the dominating factor governing the force dependence of the probability $P_{i}$ of cleavage sites to be accessible. In other words, the characteristic force scale of opening, $f_{o}$, is not directly accessible in our model as we only determine the effective force scale $f_{e} \approx f_{c} \ll f_{o}$. This is one reason why the effective force scale $f_{e}=0.06 \mathrm{pN}$ characterizing the sigmoidal behavior of $P_{i}$ in shear flow is very different compared to the force scale for unfolding induced by an external stretching force $f_{u}=1.1 \mathrm{pN}$ [13]. The other reason why the force scales are different could be that cleavage by ADAMTS13 does not require the full unfolding of the A2 domain. Only if the unfolding of the A2 domain would be a necessary step before ADAMTS13 can access the cleavage site, and only if the process of forceinduced A2 unfolding was equivalent to the shear-induced opening of the ADAMTS13 cleavage site in VWF, would $f_{u}$ correspond to the opening force scale $f_{o}$. Based on our approach modeling the shear-induced cleavage of VWF, that is close to the physiological situation, one might speculate that the process of force-induced A2 unfolding is fundamentally different. This is in line with literature results $[13,14]$ that suggest an partially unfolded, intermediate state of the A2 domain to be sufficient for ADAMTS13 cleavage. There are further conceivable mechanisms of how forces might induce increased cleavage activity in VWF. Interactions of multiple domains could, for instance, shield the cleavage site in the absence of force; only upon applied forces domains might separate and thereby enable cleavage.

Zhang et al. [13] also determined the rate of A2 refolding, employing a model where the refolding rate is described by $k_{f} \sim \exp \left(-f^{2} /(2 \kappa \mathrm{kT})\right)$. That model assumes soft compliance of the unfolded state described by a harmonic potential $U_{\text {harm }}=-f^{2} / 2 \kappa=\kappa R_{\text {ee }}^{2} / 2$, where $\kappa$ denotes the effective spring constants that characterizes the polymer elasticity and $R_{\mathrm{ee}}$ is the polymer end-to-end distance. Due to the different modeling for the closing rate, we can only compare the rates in the absence of force and thus the equilibrium constant, or equivalently the free energy difference between the two states. As a result, we obtain from our fit an estimate for the energy difference $\Delta \tilde{F}=8.5 \mathrm{kT}$, which is higher by about $2 \mathrm{kT}$ compared to the single barrier A2 unfolding and refolding kinetic model [13]. Although we would have expected a smaller free energy difference since shear presumably only partially unfolds the A2 domain, this difference is not surprising given the different model assumptions that go into the analysis of the experimental data. In terms of enzyme 
activity, Zhang et al. determined a catalytic rate constant of $\tilde{k}_{\text {cat }}=0.14 \mathrm{~s}^{-1}$ for single, accessible A2 domains and varying enzyme concentration [13]. The cleavage of A2 fragments in the absence of shear-induced unfolding is presumably a less physiological situation. Since we considered shear-induced cleavage of full-length VWF in blood plasma the substantially smaller rate constant that we obtained, $\tilde{k}_{\text {cat }}=0.0027 \mathrm{~s}^{-1}$, might not be surprising.

\section{Summary and conclusion}

In the present study we investigate the tension profile of collapsed homopolymers in shear flow by Brownian hydrodynamics simulations. Profiles for long polymers exhibit a characteristic double-peak structure that we argue is related to polymeric protrusions and forms the basis of a nucleation argument used in previous work to explain the instability mechanism behind shear-induced unfolding $[5,6]$. The range of shear rates where the double-peak structure in the tension profiles occurs roughly coincides with the peak of the variance of the chain extension, which was previously used to define the critical shear rate of unfolding. By heuristic fits of the simulation results, we find scaling relations $f_{p} \sim \dot{\gamma}^{1.6}$ and $l_{p} \sim \dot{\gamma}^{1.2}$ for the peak force and the average protrusion length, respectively. Average protrusions lengths are in the range $1<l_{p}<10$. For fixed shear rate, the protrusion length decreases with increasing globule size according to $l_{p} \sim R^{-1}$. In terms of the cohesive strength, protrusions occur for collapsed globules $\varepsilon>\varepsilon_{\text {col }}$ and for fixed shear rate the protrusion length decreases roughly linearly with $\varepsilon$ until the globule undergoes a freezing transition and protrusions disappear at about $\varepsilon \approx 3$. The peak force decreases roughly linearly both as a function of globule size and cohesive strength.

A quasi-equilibrium theory with a few fit parameters describes the behavior of the average protrusion length only in parts. This might be due to the fact that the transition between collapsed and unfolded state is slow compared to the shortest relevant time scale in the system, which is the period of globule rotation. Rare events of full polymer elongations are thus not accurately described by a quasi-equilibrium model. On the other hand, the previously established nucleation model can be successfully used to predict the critical shear rate at which unfolding sets in [5]. Since we do not observe a double-peak structure in the free draining case, we conclude that the protrusion mechanism for unfolding only applies to the case when hydrodynamic interactions are taken into account. This is an interesting observation in comparison with previous scaling arguments [5].

In the second part we consider experiments of ADAMTS13-mediated VWF cleavage. First we analyze the Morrison kinetics of the cleavage process without shear flow in denaturing buffer and obtain a Michaelis-Menten constant $K_{M}=2.3 \mathrm{nM}$ and the catalytic rate constant $\tilde{k}_{\text {cat }}=0.001 \mathrm{~s}^{-1}$. Under such denaturing conditions, we find that only a small average number of cleavage sites
$\bar{N}_{\text {open }}=0.04$ is accessible per multimer. Our results suggest domain opening to be the cleavage rate limiting factor rather than the ADAMTS13 concentration. Next we connect the simulated tension profile, via a stochastic two state model for the cleavage domain opening of each monomer, to the experimentally measured sheardependent cleavage rate of VWF in blood plasma. Due to the occurrence of protrusions one can speculate that ADAMTS13 is likely to cut VWF multimers towards the terminal ends, which are pulled out of the globules and therefore are most accessible. Our model qualitatively describes the sigmoidal increase of the cleavage rate with increasing shear rate and we obtain as a main result the parameters characterizing the probability of an individual cleavage domain to be accessible, i.e., the effective force scale $\tilde{f}_{e}=0.06 \mathrm{pN}$ and the free energy difference between open and closed state $\Delta \tilde{F}=8.5 \mathrm{kT}$. Furthermore, the catalytic rate constant of the cleavage process is found to be $\tilde{k}_{\text {cat }}=0.0027 \mathrm{~s}^{-1}$, in satisfactory agreement with $\tilde{k}_{\text {cat }}=0.001 \mathrm{~s}^{-1}$ obtained for cleavage in denaturing buffer.

The present study further elucidates the complex dynamical behavior of collapsed polymers in shear as the basis for non-equilibrium phenomena with high physiological relevance. The shear-induced VWF unfolding leads to inhomogeneous tensile force distributions and thereby strongly influences the susceptibility to proteolytic cleavage. Our findings are not limited to VWF's hemostatic function but are relevant for a number of nanotechnological and biomedical applications where functional polymers or proteins are engineered as shear-responsive smart materials or drug delivery systems. Future lines of work might involve further components that modulate the cleavage of VWF in blood plasma. It has been shown that the presence of coagulation factor VIII [39] as well as platelets [40] increases the susceptibility of VWF to cleavage by ADAMTS13. Including additional particles in the simulation model could yield valuable insight into the interplay of cofactors that bind to the polymeric monomers, conformational changes and the tension profile that in turn affects the cleavage activity. In fact, simulations of VWF and platelets have been conducted observing the formation of reversible aggregates under shear flow conditions [20]. Further VWF domain model refinements might capture atomistic details that influence the cleavage process. Domain shielding renders VWF inactive for adhesion or proteolysis [16]. On an even smaller length scale, local mutations in the VWF A2 domain that are known to affect the ADAMTS13 susceptibility [41] and also environmental conditions play a crucial role, e.g. calcium stabilizes the A2 domain and thus regulates the unfolding [42]. It would be desirable to capture such effects in simple physical models that might be able to relate mutations directly to hemostatic dysfunction.

The authors thank Alfredo Alexander-Katz, Frauke Gräter, and Matthias Schneider for stimulating discussions and acknowledge financial support from the DFG Research Unit FOR 1543 . 
Open Access This is an open access article distributed under the terms of the Creative Commons Attribution License (http://creativecommons.org/licenses/by/4.0), which permits unrestricted use, distribution, and reproduction in any medium, provided the original work is properly cited.

\section{References}

1. Alfredo Alexander-Katz, Macromolecules 47, 1503 (2014).

2. J. Evan Sadler, Annu. Rev. Biochem. 67, 395 (1998). PMID: 9759493.

3. R. Schneppenheim, U. Budde, J. Thromb. Haemost. 9, 209 (2011) PMID: 21781257.

4. T.A. Springer, J. Thromb. Haemost. 9, 130 (2011).

5. A. Alexander-Katz, M.F. Schneider, S. Schneider, A. Wixforth, R.R. Netz, Phys. Rev. Lett. 97, 138101 (2006).

6. A. Alexander-Katz, R.R. Netz, Macromolecules 41, 3363 (2008).

7. C.E. Sing, A. Alexander-Katz, Biophys. J. 98, L35 (2010).

8. Z.M. Ruggeri, Blood 108, 1903 (2006).

9. S.W. Schneider, S. Nuschele, A. Wixforth, C. Gorzelanny, A. Alexander-Katz, R.R. Netz, M.F. Schneider, Proc. Natl. Acad. Sci. U.S.A. 104, 7899 (2007).

10. Matthias Radtke, Roland Netz, Eur. Phys. J. E 37, 20 (2014).

11. C.E. Sing, J.G. Selvidge, A. Alexander-Katz, Biophys. J. 105, 1475 (2013).

12. Matthias Radtke, Roland R. Netz, Eur. Phys. J. E 38, 69 (2015).

13. X. Zhang, K. Halvorsen, C.-Z. Zhang, W.P. Wong, T.A. Springer, Science 324, 1330 (2009).

14. C. Baldauf, R. Schneppenheim, W. Stacklies, T. Obser, A. Pieconka, S. Schneppenheim, U. Budde, J. Zhou, F. Gräter, J. Thromb. Haemos. 7, 2096 (2009).

15. Hans Ulrichts, Miklós Udvardy, Peter J. Lenting, Inge Pareyn, Nele Vandeputte, Karen Vanhoorelbeke, Hans Deckmyn, J. Biol. Chem. 281, 4699 (2006) PMID: 16373331.

16. Camilo Aponte-Santamaría, Volker Huck, Sandra Posch, Agnieszka K. Bronowska, Sandra Grässle, Maria A. Brehm, Tobias Obser, Reinhard Schneppenheim, Peter Hinterdorfer, Stefan W. Schneider, Carsten Baldauf, Frauke Gräter, Biophys. J. 108, 2312 (2015).

17. Zaverio M. Ruggeri, Nat. Med. 8, 1227 (2002).

18. Shaun P. Jackson, Blood 109, 5087 (2007) PMID: 17311994.
19. H. Chen, M.A. Fallah, V. Huck, J.I. Angerer, A.J. Reininger, S.W. Schneider, M.F. Schneider, A. Alexander-Katz, Nat. Commun. 4, 1333 (2013).

20. Hsieh Chen, Alfredo Alexander-Katz, Soft Matter 9, 10381 (2013).

21. J.E. Sadler, Blood 112, 11 (2008).

22. Han-Mou Tsai, Int. J. Hematol. 91, 1 (2010).

23. Volker Huck, Matthias F. Schneider, Christian Gorzelanny, Stefan W. Schneider, Thromb. Haemost. 111, 598 (2014) PMID: 24573248

24. M. Furlan, Ann. Hematol. 72, 341 (1996) PMID: 8767102.

25. Svenja Lippok, Tobias Obser, Jochen P. Müller, Valentin K. Stierle, Martin Benoit, Ulrich Budde, Reinhard Schneppenheim, Joachim O. Rädler, Biophys. J. 105, 1208 (2013).

26. J.E. Sadler, Proc. Natl. Acad. Sci. U.S.A. 99, 11552 (2002).

27. J.-f. Dong, Blood 100, 4033 (2002).

28. Weiqiang Gao, Patricia J. Anderson, Elaine M. Majerus, Elodee A. Tuley, J. Evan Sadler, Proc. Natl. Acad. Sci. U.S.A. 103, 19099 (2006) PMID: 17146059 PMCID: PMC1681350.

29. Junyi Ying, Yingchen Ling, Lisa A. Westfield, J. Evan Sadler, Jin-Yu Shao, Biophys. J. 98, 1685 (2010).

30. Svenja Lippok, Matthias Radtke, Tobias Obser, Lars Kleemeier, Reinhard Schneppenheim, Ulrich Budde, Roland R. Netz, Joachim O. Rädler, Biophys. J. 110, 545 (2016).

31. Jens Rotne, Stephen Prager, J. Chem. Phys. 50, 4831 (1969).

32. Hiromi Yamakawa, J. Chem. Phys. 53, 436 (1970).

33. Theo GM Van de Ven, Colloidal hydrodynamics (Academic Press, 1989).

34. J.F. Morrison, Biochim. Biophys. Acta Enzymol. 185, 269 (1969).

35. T.R. Einert, C.E. Sing, A. Alexander-Katz, R.R. Netz, Eur. Phys. J. E 34, 130 (2011).

36. W.E. Fowler, L.J. Fretto, K.K. Hamilton, H.P. Erickson, P.A. McKee, J. Clin. Investig. 76, 1491 (1985)

37. I. Singh, H. Shankaran, M.E. Beauharnois, Z. Xiao, P. Alexandridis, S. Neelamegham, J. Biol. Chem. 281, 38266 (2006).

38. Yan-Feng Zhou, Edward T. Eng, Noritaka Nishida, Chafen Lu, Thomas Walz, Timothy A. Springer, EMBO J. 30, 4098 (2011).

39. W. Cao, S. Krishnaswamy, R.M. Camire, P.J. Lenting, X.L. Zheng, Proc. Natl. Acad. Sci. U.S.A. 105, 7416 (2008).

40. C.G. Skipwith, W. Cao, X.L. Zheng, J. Biol. Chem. 285, 28596 (2010).

41. A.J. Xu, T.A. Springer, J. Biol. Chem. 288, 6317 (2013).

42. Arjen J. Jakobi, Alireza Mashaghi, Sander J. Tans, Eric G. Huizinga, Nat. Commun. 2, 385 (2011). 